\title{
Effekte auf PSA-Langzeitkontrolle
}

- Mit einem Anteil von rund $26 \%$ steht das Prostatakarzinom (PCa) an erster Stelle bei den zum Tode führenden Krebserkrankungen. Umso höher ist der Stellenwert wirksamer und gut verträglicher Therapieoptionen. Seit über einem Jahr hat sich der GnRH-Blocker Degarelix (Firmagon $^{\circledR}$ ) in der P(a-Therapie bewährt. Auf dem 4. North Eastern European Meeting (NEEM) in Riga wurden nun Langzeitdaten einer noch laufenden Extensionsanalyse mit einem Follow-up von 3,5 Jahren der Zulassungsstudie von Degarelix präsentiert. Alle Patienten hatten nach der zwölfmonatigen Zulassungsstudie die Möglichkeit, sich weiter mit Degarelix behandeln zu lassen. Die Umsteiger von Leuprorelin auf Degarelix wurden auf die Erhaltungsdosen $160 \mathrm{mg}$ oder $80 \mathrm{mg}$ randomisiert. Die Zeitspanne bis zum PSA-Versagen oder
Tod war dabei bei Patienten unter Degarelix signifikant länger als unter Leuprorelin $7,5 \mathrm{mg}(p=0,0495)$. Auch bei Patienten, die sekundär nach einem Jahr Degarelix erhielten, nahm bei einem medianen Follow-up von 27,5 Monaten das Risiko, eine PSA-Progression oder den Tod zu erleiden, signifikant $a b(p=0,003)$. Dies traf auch für die Subgruppe mit PSA-Ausgangswerten $\geq$ $20 \mathrm{ng} / \mathrm{ml}$ in Bezug auf die PSA-Progression zu.

Die Daten bestätigen die Ergebnisse der Zulassungsstudie und unterstützen die Anwendung von Degarelix als First-LineTherapie bei der Androgendeprivationstherapie.

Nach Informationen von

Ferring, Kiel

\section{Cabazitaxel schließt therapeutische Lücke}

\section{_ Sind Patienten mit metastasiertem ka-} strationsresistentem Prostatakarzinom (mKRPC) nach First-Line-Therapie mit Docetaxel progredient, wird mit dem Taxan Cabazitaxel in Kombination mit Prednison künftig erstmals eine Zweitlinientherapie zur Verfügung stehen, für die ein Überlebensvorteil nachgewiesen ist. Erst kürzlich wurde Cabazitaxel in den USA in einem beschleunigten Zulassungsprüfverfahren für die Zweitlinientherapie nach Docetaxel zugelassen. Die Zulassung von Cabazitaxel wird eine wichtige therapeutische Lücke schließen, erläuterte Dr. med. Götz Geiges, Berlin. Auch in Europa ist der Zulassungsantrag für Cabazitaxel gestellt. In den Therapieempfehlungen der Europäischen Gesellschaft für Urologie wird die Substanz bereits als Zweitlinientherapie nach Docetaxel-Vorbehandlung mit Evidenzlevel A empfohlen. Mit der US-Zulassung ist die Kombination Cabazitaxel (Jevtana ${ }^{\circledR}$ ) plus Prednison dort die erste und derzeit einzige zugelassene Zweitlinientherapie für Patienten mit mKRPC nach Docetaxel-haltiger Erstlinientherapie, betonte Geiges. Die in dieser Situation häufig eingesetzte Kombination Mitoxantron/Prednison (MP) ist nur zur Schmerzlinderung bei mKRPC Patienten zugelassen, die auf Analgetika nicht mehr ansprechen und bei denen eine Strahlentherapie nicht indiziert ist.

In der TROPIC-Studie-eine randomisierte Phase-III-Studie, an der 755 mit Docetaxelvorbehandelte mKRPC-Patienten teilnahmen - reduzierte Cabazitaxel/Prednison das relative Sterberisiko statistisch signifikant um 30\% (HR: 0,70; $p<0,0001$ ) gegenüber dem MP-Regime. Die mittlere Gesamtüberlebenszeit betrug im Cabazitaxel-Arm 15,1 Monate versus 12,7 Monate im MP-Arm. Der mittlere Überlebensvorteil bestätigte sich in allen untersuchten Subgruppen unabhängig vom Alter und Allgemeinzustand der Patienten sowie der Vorbehandlung.

Der statistisch signifikante mittlere Überlebensvorteil, den Cabazitaxel in der TROPIC-Studie erreichte, wurde nicht durch unvorhersehbare Nebenwirkungen überschattet. Im Vordergrund standen - wie von den Taxanen bekannt - hämatologische Nebenwirkungen. Schwere (Grad 3-4) nicht-hämatologische Nebenwirkungen waren: Durchfall 6,2\%, Übelkeit 1,9\% und Erbrechen 1,9\%.

Nach Informationen von

Sanofi-Aventis, Frankfurt am Main

\section{Fesoterodin besser als sein Vorgänger}

Auf dem 62. Kongress der Deutschen Gesellschaft für Urologie in Düsseldorf wurden die Ergebnisse der beiden größten placebokontrollierten randomisierten Head-to-Head-Studien zum direkten Vergleich zweier Antimuskarinika vorgestellt. Fesoterodin $8 \mathrm{mg}\left(\right.$ Toviaz $\left.^{\circledR}\right)$ erwies sich in der FACT- und der FACT-2-Studie dem bisherigen Goldstandard Tolterodin retard $4 \mathrm{mg}$ (Detrusitol ${ }^{\circledR}$ ) und Placebo überlegen in der Behandlung der Symptome der überaktiven Blase. Signifikante Unterschiede zeigten sich sowohl in Bezug auf die Verbesserung des primären Endpunkts und der Reduktion der Dranginkontinenzepisoden als auch in der Trockenheitsrate. In beiden Studien spiegelten sich diese Ergebnisse auch in den von Patienten geäußerten Verbesserungen ihres Blasenzustandes wider.

Nach Informationen von Pfizer, Berlin

\section{Cranberry-Präparat für eine gesunde Blase}

In den nordamerikanischen Cranberrys (Vaccinium macrocarpon) sind Proanthocyanidine vom Typ A (PAC) enthalten. Proanthocyanidine haben aufgrund ihrer Anti-Adhäsionskräte, die ein Anheften an und somit ein Eindringen von Bakterien in die Blasenwand verhindern, eine harnwegsschützende Wirkung. Für einen zuverlässigen Effekt ist eine tägliche Dosierung von 36 mg PAC notwendig. In Deutschland erfüllt nur das Präparat ellura ${ }^{\circledR}$ diesen Anspruch. Das Cranberry-Präparat zeichnet sich zudem durch eine maximale Bioaktivität seiner Wirkstoffe aus.

Nach Informationen von Lucien Ortscheit, Saarbrücken

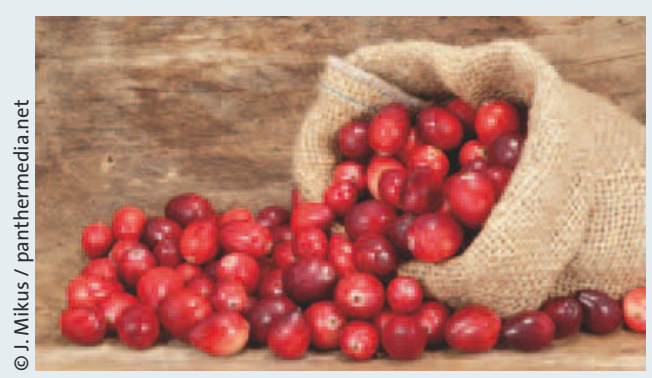

